\title{
Dynamical Coulomb Blockade and the Derivative Discontinuity of Time-Dependent Density Functional Theory
}

\author{
S. Kurth, ${ }^{1,2,3}$ G. Stefanucci, ${ }^{4,3}$ E. Khosravi, ${ }^{5,3}$ C. Verdozzi, ${ }^{6,3}$ and E. K. U. Gross ${ }^{5,3}$ \\ ${ }^{1}$ Nano-Bio Spectroscopy Group, Departamento de Física de Materiales, Universidad del País Vasco UPV/EHU, \\ Centro Física de Materiales CSIC-UPV/EHU, Avenida Tolosa 72, E-20018 San Sebastián, Spain \\ ${ }^{2}$ IKERBASQUE, Basque Foundation for Science, E-48011 Bilbao, Spain \\ ${ }^{3}$ European Theoretical Spectroscopy Facility (ETSF) \\ ${ }^{4}$ Dipartimento di Fisica, Università di Roma Tor Vergata, Via della Ricerca Scientifica 1, 00133 Rome, Italy \\ ${ }^{5}$ Max-Planck Institut für Mikrostrukturphysik, Weinberg 2, D-06120 Halle, Germany \\ ${ }^{6}$ Mathematical Physics, Lund University, 22100 Lund, Sweden
}

(Received 19 November 2009; published 7 June 2010)

\begin{abstract}
The role of the discontinuity of the exchange-correlation potential of density functional theory is studied in the context of electron transport and shown to be intimately related to Coulomb blockade. By following the time evolution of an interacting nanojunction attached to biased leads, we find that, instead of evolving to a steady state, the system reaches a dynamical state characterized by correlation-induced current oscillations. Our results establish a dynamical picture of Coulomb blockade manifesting itself as a periodic sequence of charging and discharging of the nanostructure.
\end{abstract}

Coulomb blockade (CB) [1] is one of the true hallmarks of electron-electron interactions in mesoscopic and nanoscale physics. In molecular transport, $\mathrm{CB}$ is due to an electrostatic barrier induced by the electrons in the device which prevents further electrons from tunneling in [2]. Progress in the theoretical description and experimental manipulation of $\mathrm{CB}$ is expected to foster advances in nanoelectronics and quantum information technology [3-5]. On the theoretical side, many aspects of $\mathrm{CB}$ can be understood in a rather simple way [6]. However, to achieve quantitative accuracy in real systems, an ab initio description is required, something which has not been accomplished yet.

In fact, most current $a b$ initio treatments of transport are limited to the steady-state regime and based on the Landauer formalism combined with density functional theory (DFT) [7]. Within this prescription ( + DFT), the agreement with experiment is often poor, particularly for devices weakly coupled to leads. The approach has also been criticized on fundamental theoretical grounds (see, e.g., [8]). A main reason for its failure has been identified in the shortcomings of typical exchange-correlation (XC) functionals of static DFT [9], such as, e.g., the lack of a derivative discontinuity in popular local density approximations (LDA) and generalized gradient approximations [10]. The importance of the derivative discontinuity in a static picture of $\mathrm{CB}$ for finite systems has been investigated in Ref. [11].

As transport is an intrinsically nonequilibrium phenomenon, the scientific community has progressively shifted to time-dependent (TD) approaches in recent years (see, e.g., Refs. [12,13]). This shift also recognizes the fact that significant novel physics occurs in the time domain. While different approaches to TD transport have been developed, time-dependent DFT (TDDFT) [14] offers a computationally efficient but still in principle exact method [15].

In relation to $\mathrm{CB}$, it is then quite natural to address the following issues: (i) What is the connection between a TDDFT description of $\mathrm{CB}$ in real time and the one of standard steady-state approaches? (ii) Which features an approximate XC functional should have to describe CB?

This Letter takes a first step in answering these basic questions. We present here a TDDFT study of CB in the time domain for a correlated single-level quantum dot (QD) weakly coupled to leads. We use a novel XC functional [16] exhibiting the proper derivative discontinuity and find that the assumption that the system evolves towards a steady state is not generally justified. In contrast, the discontinuity of the potential leads to self-sustained oscillations induced by electron correlations, with historydependent frequencies and amplitudes. Our results thus reveal dynamical aspects of $\mathrm{CB}$, which are not accessible by traditional steady-state approaches.

The system and its TDDFT time evolution.-The Hamiltonian for a single-level QD coupled to two semiinfinite, noninteracting $1 \mathrm{D}$ leads is

$$
\hat{H}(t)=\hat{H}_{\mathrm{QD}}+\sum_{\alpha=L, R} \hat{H}_{\alpha}+\hat{H}_{T}+\hat{H}_{\text {bias }}(t) .
$$

In Eq. (1), $\hat{H}_{\alpha}=-\sum_{\sigma} \sum_{i=1}^{\infty}\left(V \hat{c}_{i+1 \alpha, \sigma}^{\dagger} \hat{c}_{i \alpha, \sigma}+\right.$ H.c.), and $\hat{H}_{T}=-\sum_{\alpha, \sigma}\left(V_{\text {link }} \hat{c}_{1 \alpha, \sigma}^{\dagger} \hat{c}_{0 \sigma}+\right.$ H.c. $)$, respectively, describe, in standard notation, the leads and their coupling to the QD. $\hat{H}_{\alpha}$ and $\hat{H}_{T}$ contain only nearest neighbor hopping terms: $V$ (in the left, $L$, and right, $R$, lead) and $V_{\text {link }}$ (to/from the QD). In the following, we take both $V$ and $V_{\text {link }}$ to be positive. The effect of applying a TD bias 
is contained in $\hat{H}_{\text {bias }}(t)=\sum_{\alpha, \sigma} \sum_{i=1}^{\infty} W_{\alpha}(t) \hat{c}_{i \alpha, \sigma}^{\dagger} \hat{c}_{i \alpha, \sigma}$. Finally, the QD Hamiltonian is

$$
\hat{H}_{\mathrm{QD}}=v_{\mathrm{ext}} \sum_{\sigma} \hat{n}_{0 \sigma}+U \hat{n}_{0 \uparrow} \hat{n}_{0 \downarrow}
$$

with $\hat{n}_{0 \sigma}=\hat{c}_{0 \sigma}^{\dagger} \hat{c}_{0 \sigma}$ the density for electrons with spin $\sigma$. Two parameters describe the QD: the charging energy $U$ and a static gate voltage $v_{\text {ext }}$. For time $t \leq 0$, the system is in equilibrium; at $t>0$, a bias $W_{\alpha}(t)$ is applied.

Within TDDFT, the many-body Hamiltonian (1) is mapped onto an effective one-particle Kohn-Sham (KS) Hamiltonian. The KS dynamics produces the exact TD density, provided we know the exact KS potential whose density dependence is nonlocal in space and time. In practice, one has to resort to approximations. As shown below, we can already gain significant insight via an approximate, adiabatic KS potential. The approximation we use is based on a ground state $\mathrm{XC}$ functional obtained from an LDA of the nonuniform 1D Hubbard model, via the Bethe ansatz (BALDA) [16]. In the context of TDDFT, an adiabatic version of this functional (ABALDA) has already been used to investigate the dynamics of Hubbard clusters [17]. Within the ABALDA, the KS Hamiltonian reads $\hat{H}_{\mathrm{KS}}(t)=\hat{H}_{\mathrm{QD}, \mathrm{KS}}(t)+\sum_{\alpha=L, R} \hat{H}_{\alpha}+\hat{H}_{T}+\hat{H}_{\text {bias }}(t)$, with

$$
\hat{H}_{\mathrm{QD}, \mathrm{KS}}(t)=\sum_{\sigma} v_{\mathrm{KS}}\left[n_{0}(t)\right] \hat{n}_{0 \sigma} .
$$

The KS potential is a functional of the instantaneous density $n_{0}(t)=\sum_{\sigma} n_{0 \sigma}(t)$ on the QD. Explicitly,

$$
v_{\mathrm{KS}}\left[n_{0}(t)\right]=v_{\mathrm{ext}}+\frac{1}{2} U n_{0}(t)+v_{\mathrm{xc}}\left[n_{0}(t)\right] .
$$

The original BALDA functional was devised for a uniform chain with Hubbard interaction $U$ and nearest neighbor hopping $V$ : in this case, the ratio $U / V$ is the relevant parameter in $v_{\mathrm{xc}}$ [16]. However, in the CB regime, which we wish to address here, the electrons need to be largely localized in the device. For our model system, this corresponds to (i) taking $V_{\text {link }}$ significantly smaller than $V$ and (ii) using $U / V_{\text {link }}$ as relevant parameter in $v_{\mathrm{xc}}$. Accordingly, we propose the functional form

$$
\begin{aligned}
& v_{\mathrm{xc}}[n]=\theta(1-n) v_{\mathrm{xc}}^{(<)}[n]-\theta(n-1) v_{\mathrm{xc}}^{(<)}[2-n], \\
& v_{\mathrm{xc}}^{(<)}[n]=-\frac{1}{2} U n-2 V_{\operatorname{link}}\left[\cos \left(\frac{\pi n}{2}\right)-\cos \left(\frac{\pi n}{\beta}\right)\right],
\end{aligned}
$$

where the parameter $\beta$ is determined by the condition

$$
\frac{2 \beta}{\pi} \sin (\pi / \beta)=4 \int_{0}^{\infty} d x \frac{J_{0}(x) J_{1}(x)}{x\left[1+\exp \left(U x /\left(2 V_{\text {link }}\right)\right)\right]}
$$

with $J_{i=0,1}(x)$ Bessel functions. For $V_{\text {link }} \rightarrow 0, v_{\text {xc }}[n] \rightarrow$ $-\theta(1-n) U n / 2+\theta(n-1) U(2-n) / 2$, which correctly reproduces the exact XC potential of the isolated QD. This $v_{\text {xc }}$ also leads to densities (not shown) in good agreement with the quantum Monte Carlo data [18] for the Anderson model. At half-filling, $v_{\mathrm{xc}}[n]$ is discontinuous [19]: $v_{\text {xc }}\left[1^{+}\right]-v_{\text {xc }}\left[1^{-}\right]=U-4 V_{\text {link }} \cos \left(\frac{\pi}{\beta}\right)$. The exact XC potential is certainly discontinuous for the 1D Hubbard model or for the isolated QD. However, for the QD connected to noninteracting leads it is reasonable to expect a small smoothening of the discontinuity (this is supported by preliminary calculations, not shown here, of the exact $v_{\mathrm{xc}}$ for an Anderson impurity in small clusters). We therefore modified the discontinuous (at $n=1) v_{\text {xc }}[n]$ of Eq. (5), with a softened, continuous version $\tilde{v}_{\mathrm{xc}}(n)=$ $f(n) v_{\mathrm{xc}}^{(<)}[n]-(1-f(n)) v_{\mathrm{xc}}^{(<)}[2-n] . \quad$ Here, $\quad f(n)=$ $\frac{1}{\exp ((n-1) / a)+1}$, with a smoothening parameter $a$. The smoothening of the KS potential also has the advantage of alleviating the numerical difficulties caused by the sudden changes of $v_{\mathrm{xc}}$ during time propagation.

To propagate the lead-dot-lead KS system in time, we use a recently developed TD algorithm [20] for an open system of (effectively) noninteracting electrons, as required in TDKS. In all simulations below, energies are measured in units of $V$, times in units of $V^{-1}$ and currents in units of $|e| V$, where $e$ is the charge of the carriers. The sharp slope near $n=1$ in $v_{\text {xc }}$ has a profound impact on the time evolution of the density on the QD as well as on the current through it.

$T D$ transport in the $C B$ regime.-In Fig. 1, we show the time evolution of the density, the current and the KS potential with a smoothening parameter $a=10^{-4}$ for three bias values and $V_{\text {link }}=0.3, v_{\text {ext }}=2, U=2$, and Fermi energy $\varepsilon_{\mathrm{F}}=1.5$ (as shown below, this choice of the parameters corresponds to the $\mathrm{CB}$ regime). The system is in its ground state for $t<0$; the equilibrium density is calculated self-consistently with the $v_{\mathrm{KS}}$ of Eq. (4) but in static DFT. At $t=0$, a dc bias $W_{L}$ is suddenly switched on in the

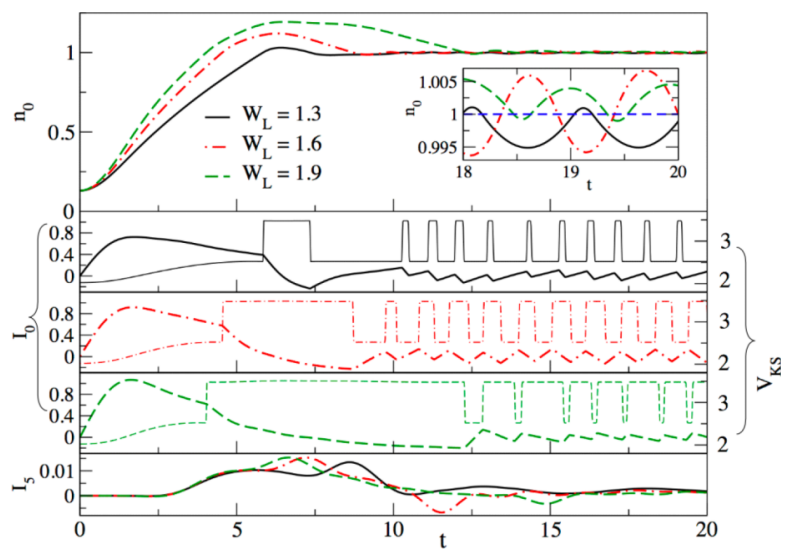

FIG. 1 (color online). Time evolution of the density, current, and KS potential for three different biases. In all panels, the solid, chain, and dashed lines refer to $W_{L}=1.3,1.6,1.9$, respectively. Top panel: densities at the QD. The inset shows the density at the end of the propagation period. Middle three panels: current through the QD (thick lines) and KS potential (thin lines). Bottom panel: current five sites away from the QD. 
left lead. Remarkably, for this set of parameters and within a certain range of biases, the system does not evolve towards a steady state; instead, after a transient period, we see self-sustained density oscillations around unity (Fig. 1, top panel), with an amplitude of the order of $10^{-3}$ (inset in the same panel). For the lower bias, $W_{L}=$ 1.3 , the density remains below unity for most of the period, for $W_{L}=1.6$ the density roughly oscillates around 1 , and for $W_{L}=1.9$ the density exceeds unity for most of the period. The oscillations are also present in the current (middle three panels), but this time with a much larger amplitude relative to its average value. The saw-tooth structure is consistent with the continuity equation (the density is approximately piecewise parabolic). Note that away from the QD, the oscillations in the current (and in the density) disappear (bottom panel). Oscillations are clearly visible also in $v_{\mathrm{KS}}$ (middle three panels). Because of the fairly large jump in $v_{\mathrm{xc}}$ and the small amplitude of the density oscillations, the KS potential is a train of almost rectangular pulses. For larger biases, the density remains above unity for longer times, and the width of the pulses in $v_{\mathrm{KS}}$ extends in time. The oscillations discussed here are a direct consequence of the discontinuity in $v_{\mathrm{xc}}$. For similar calculations (not shown) in the Hartree approximation, where $v_{\mathrm{KS}}$ is continuous in the density, the system does evolve towards a steady state.

History dependence, oscillations, and smoothening.We have calculated the time evolution of the system as obtained by switching the bias as $W_{L}(t)=W_{L} \sin ^{2}\left(\frac{\pi t}{2 T_{\text {switch }}}\right)$ for $t<T_{\text {switch }}$ and $W_{L}(t)=W_{L}=$ const otherwise. The Fourier analysis of $n_{0}(t)$ reveals peaks whose position and height depend on $T_{\text {switch }}$, i.e., on the history of the applied bias. We found that for any small but finite $a$, the amplitude of the oscillations approaches zero as $T_{\text {switch }} \rightarrow$ $\infty$. We therefore conclude that in this case the L + DFT approach gives the same solution as TDDFT for sufficiently slow switching [21]. For $a=0$, instead, the system never reaches a steady state.

$C B$ regime from the steady-state approach.-The oscillations just described are distinct from those occurring in the presence of single particle bound states [24,25]: They are induced by electron correlations, i.e., are absent in the mean field (Hartree) approximation. An especially revealing feature is that at long times the system is in a dynamical state of oscillating density and current, whose time average is fairly constant for a large range of biases. For further insight, we now use the L + DFT approach which, as said above, yields the same solution as TDDFT for $a \neq 0$ and for adiabatic switching. Using nonequilibrium Green's function techniques, the value $n^{\infty}$ of the steady-state density at the QD is given by the self-consistency condition [26]

$$
n^{\infty}=2 \sum_{\alpha=L, R} \int_{-\infty}^{\varepsilon_{F}+W_{\alpha}} \frac{d \omega}{2 \pi} \Gamma\left(\omega-W_{\alpha}\right)|G(\omega)|^{2}
$$

Here $G(\omega)=\left(\omega-v_{\mathrm{KS}}\left[n^{\infty}\right]-\sum_{\alpha} \Sigma\left(\omega-W_{\alpha}\right)\right)^{-1}$ is the retarded Green's function at the QD site, which depends on $n^{\infty}$ through $v_{\mathrm{KS}}, \Sigma(\omega)$ is the embedding self-energy of the leads, and the width function $\Gamma(\omega)=-2 \operatorname{Im}[\Sigma(\omega)]$.

We first consider a discontinuous $v_{\text {xc }}$ (i.e., $\left.a=0\right)$ ). In Fig. 2, left panel, we display the left-hand side (lhs) and the right-hand side (rhs) of Eq. (8) as functions of $n^{\infty}$ for the parameters $V=1, V_{\text {link }}=0.3, U=2, v_{\text {ext }}=2, \varepsilon_{F}=1.5$, and for different values of the dc bias $W_{L}$. These are the parameters used in our TD simulations of Fig. 1. Interestingly, there is a range of applied bias voltages for which Eq. (8) does not have a solution. Since Eq. (8) is a condition for the QD density at the steady state, it follows that, as a direct consequence of the discontinuity in $v_{\mathrm{xc}}$, for some values of $W_{\alpha}$, no steady state exists. On the other hand, for $a=10^{-4}$, as in our TD simulations, Eq. (8) admits steady-state solutions; however, from Fig. 1, we know that, in general, the system time evolves towards an oscillating regime and that the solution of the L + DFT scheme is a solution in TDDFT for adiabatic switching.

To show that in fact we are in the $\mathrm{CB}$ regime, in Fig. 2, right panel, we display the steady-state density $n^{\infty}$, as a function of $W_{L}$. As before, $v_{\mathrm{ext}}=2, \varepsilon_{\mathrm{F}}=1.5, U=2$, and $a=10^{-4}$. We see a plateau in the density at $n=1$ developing for a range of $W_{L}$. The plateau increases with decreasing $V_{\text {link }}$, and in the limit of small $V_{\text {link }}$ its length becomes equal to the charging energy $U$. This is the usual CB picture: if the site is occupied by one electron, a second electron can only jump in if its energy exceeds the charging energy of the QD. For $V_{\text {link }}=0.3$, the $W_{L}$ 's of Fig. 1 correspond to the beginning, middle, and end of the density plateau. Our results show that there exists a range of biases, starting at a critical value $W_{c}$, for which the decay time of the oscillations becomes infinite.

Some considerations on $v_{\mathrm{xc}}$ and the ABALDA.Dropping only the assumption of locality in space, $v_{\mathrm{xc}}$ will depend on the instantaneous densities at few, say $M$, sites around the QD. This gives a steady-state condition similar to Eq. (8), in the form of a set of equations for the densities at the $M$ sites. For a discontinuous $v_{\text {xc }}$ at one (or more) of the $M$ sites, these equations may not have a
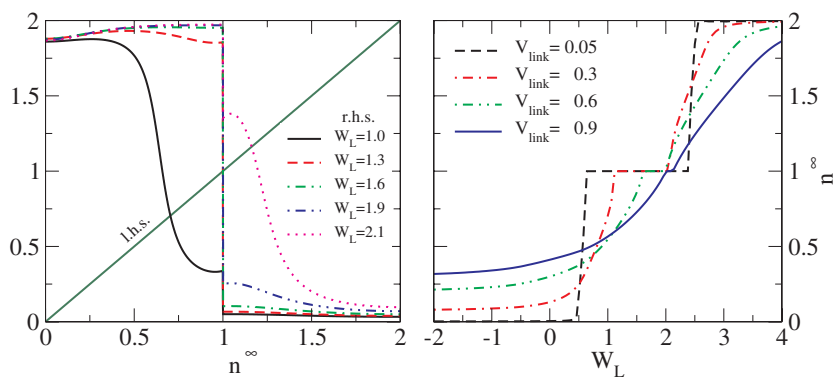

FIG. 2 (color online). Left panel: Graphical solution of Eq. (8) for few values of the applied bias $W_{L}$. Right panel: Steady-state density as function of $W_{L}$ for a smoothened $v_{\mathrm{KS}}\left(a=10^{-4}\right)$ and few values of the dot-lead hopping $V_{\text {link }}$. 
solution, similar to what we found in Fig. 2. The role of nonadiabaticity is much harder to assess since it requires the development of XC functionals with memory, e.g., via nonequilibrium many-body perturbation theory $[13,27,28]$ or fluid dynamical considerations [29,30].

We can now provide an answer to the two questions posed at the beginning of this Letter. A discontinuous or rapidly varying $v_{\mathrm{xc}}$ is central to the description of $\mathrm{CB}$ within L + DFT and TDDFT. For KS potentials with a true discontinuity, the steady-state self-consistency condition in L + DFT cannot always be satisfied. Considering a smoothened discontinuity (which, as said earlier, is physically more realistic) the L + DFT approach yields a clearcut $\mathrm{CB}$ scenario. However, the very same XC potential used in a TDDFT framework leads to a dynamical state with history-dependent, self-sustained density and current oscillations: where the static approach gives $\mathrm{CB}$, the TD approach gives oscillations. The average of these oscillations corresponds to the L + DFT solution. The latter is a TDDFT solution only for an adiabatic switch on.

To conclude, our results suggest that in a transport setup CB manifests itself as an intrinsically TD phenomenon; i.e., it corresponds to an oscillatory current representing the intuitive picture of $\mathrm{CB}$ as a sequence of charging and discharging of the weakly coupled molecule or QD. While our calculations are performed for a model consisting of a single correlated QD coupled to noninteracting leads, we expect the results to be of general nature: In the continuum-real-space TDDFT description, whenever the particle number on the molecule or QD crosses an integer, the discontinuity of the time-dependent XC potential [31] will trigger persisting charge and current oscillations, which cannot be captured in any steady-state approach.

We would like to acknowledge useful discussions with César Proetto. S. K., C. V., and E. K. U. G. acknowledge the hospitality of KITP where part of this work was carried out. S.K. acknowledges funding by the "Grupos Consolidados UPV/EHU del Gobierno Vasco" (IT-31907). C. V. is supported by ETSF (INFRA-2007-211956). This research was supported in part by the National Science Foundation under Grant No. PHY05-51164.

[1] C. J. Gorter, Physica (Amsterdam) 17, 777 (1951).

[2] I. O. Kulik and R. I. Shekhter, Zh. Eksp. Teor. Fiz. 68, 623 (1975) [Sov. Phys. JETP 41, 308 (1975)].

[3] See, e.g., Single Charge Tunneling: Coulomb Blockade Phenomena in Nanostructures, edited by H. Grabert and M. H. Devoret, NATO ASI Series B (Plenum, New York, 1992), p. 234.
[4] M. di Ventra, Electrical Transport in Nanoscale Systems (Cambridge University Press, Cambridge, England, 2008).

[5] See, e.g., K. Phoa et al., Nano Lett. 9, 3225 (2009); S. Gustavsson et al., Surf. Sci. Rep. 64, 191 (2009).

[6] C. W. J. Beenakker, Phys. Rev. B 44, 1646 (1991).

[7] N. D. Lang, Phys. Rev. B 52, 5335 (1995); J. Taylor et al., Phys. Rev. B 63, 245407 (2001); M. Brandbyge et al., Phys. Rev. B 65, 165401 (2002); F. Evers et al., Phys. Rev. B 69, 235411 (2004).

[8] M. Koentopp et al., J. Phys. Condens. Matter 20, 083203 (2008).

[9] P. Schmitteckert and F. Evers, Phys. Rev. Lett. 100, 086401 (2008).

[10] C. Toher et al., Phys. Rev. Lett. 95, 146402 (2005).

[11] K. Capelle et al., Phys. Rev. Lett. 99, 010402 (2007).

[12] R. Baer et al., J. Chem. Phys. 120, 3387 (2004); K. Burke et al., Phys. Rev. Lett. 94, 146803 (2005); C. Verdozzi et al., Phys. Rev. Lett. 97, 046603 (2006); C. L. Cheng et al., Phys. Rev. B 74, 155112 (2006); X. Zheng et al., Phys. Rev. B 75, 195127 (2007); P. Bokes et al., Phys. Rev. Lett. 101, 046402 (2008); F. Heidrich-Meisner et al., Phys. Rev. B 79, 235336 (2009); V. Moldoveanu et al., New J. Phys. 11, 073019 (2009).

[13] P. Myöhänen et al., Europhys. Lett. 84, 67001 (2008); Phys. Rev. B 80, 115107 (2009).

[14] E. Runge and E. K. U. Gross, Phys. Rev. Lett. 52, 997 (1984).

[15] G. Stefanucci and C.-O. Almbladh, Europhys. Lett. 67, 14 (2004); Phys. Rev. B 69, 195318 (2004).

[16] N. A. Lima et al., Phys. Rev. Lett. 90, 146402 (2003).

[17] C. Verdozzi, Phys. Rev. Lett. 101, 166401 (2008).

[18] X. Wang et al., Phys. Rev. B 77, 045119 (2008).

[19] N. A. Lima et al., Europhys. Lett. 60, 601 (2002).

[20] S. Kurth et al., Phys. Rev. B 72, 035308 (2005).

[21] The steady-state solution achieved in TDDFT with an adiabatically switched bias is the same as the L + DFT one only for adiabatic approximations to $v_{\mathrm{xc}}[15,22,23]$.

[22] M. Koentopp et al., Phys. Rev. B 73, 121403(R) (2006).

[23] G. Stefanucci et al., in Molecular and Nano Electronics: Analysis, Design, and Simulation, edited by J. Seminario (Elsevier, Amsterdam, 2006).

[24] G. Stefanucci, Phys. Rev. B 75, 195115 (2007).

[25] E. Khosravi et al., Appl. Phys. A 93, 355 (2008); Phys. Chem. Chem. Phys. 11, 4535 (2009).

[26] Another term must be added on the rhs of Eq. (8) if the asymptotic $t \rightarrow \infty$ Hamiltonian has split-off eigenstates [24,25].

[27] M. Puig von Friesen, C. Verdozzi, and C.-O. Almbladh, Phys. Rev. Lett. 103, 176404 (2009).

[28] K. S. Thygesen, Phys. Rev. Lett. 100, 166804 (2008).

[29] J. F. Dobson et al., Phys. Rev. Lett. 79, 1905 (1997).

[30] I. V. Tokatly, Phys. Rev. B 75, 125105 (2007), and references therein.

[31] M. Lein and S. Kümmel, Phys. Rev. Lett. 94, 143003 (2005). 